\title{
Classic Kaposi's Sarcoma Showing Multiple Organ Involvement: A Case Report and Brief Review of Literature
}

\author{
Nilüfer ONAK KANDEMIR,, Figen BARUT,, ${ }^{2}$ Gamze YURDAKAN,, ${ }^{2}$ Banu DOĞAN GÜN, ${ }^{2}$ İsmail Eren BíROL, ${ }^{2}$ \\ Şükrü Oğuz ÖZDAMAR²
}

'Department of Medical Pathology, Ankara Atatürk Training and Research Hospital, Ankara-Turkey

${ }^{2}$ Department of Medical Pathology, Bülent Ecevit University Faculty of Medicine, Zonguldak-Turkey

\begin{abstract}
SUMMARY
Kaposi's sarcoma (KS) is a vascular tumor with different epidemiological, clinical, and morphologic features, and KS-associated herpes virus plays role in its etiology. In the pathogenesis of these tumors, inflammatory, immunological, and oncogenic factors are closely related to each other. Classic KS is characterized by cutaneous lesions and mild clinical course. However, with the addition of various factors to the disease process, KS lesions can occur in unusual locations and cause unexpected clinical symptoms. In this study, we present a case of gastrointestinal system involvement in KS and lymphadenopathic KS following interstitial lung disease and steroid treatment in a 72-year-old male patient who was treated for cutaneous classic KS for 10 years. Histopathologic differential diagnosis of KS lesions seen in unusual localizations is discussed in the context of literature.
\end{abstract}

Keywords: Gastrointestinal system; Kaposi’s sarcoma; lymphadenopathy.

Copyright $\odot$ 2017, Turkish Society for Radiation Oncology

\section{Introduction}

Kaposi's sarcoma (KS) is a vascular tumor in which KSassociated herpes virus (KSHV/HHV-8) plays a role in its etiology and has four different epidemiological forms (classic KS, African-endemic KS, iatrogenic KS, and AIDS-related KS).

$\mathrm{KSHV}$, a member of the gamma herpes virus family, is the etiological agent of the disease but is not capable of causing KS alone. Although it is not fully clear which factors trigger the oncogenic transformation of KSHV, iatrogenic or acquired immunosuppression appears to be an important factor for KS development. The oncogenic genes of KSHV induce genes that stimulate cellular proliferation, transformation, cellular signaling, cytokine production, immune escape, antiapoptotic, and angiogenenic action. Although the origin of spindle cells, the real neoplastic cells of KS, is still contro- versial, it has been shown that they develop from uncommitted endothelial progenitors, with the lymphatic endothelial type being most predominant.[1]

KS is a neoplastic model where cancer and inflammation are intertwined and has different epidemiological forms with variable clinical course despite a common etiological agent. Classic KS is usually characterized by slowly progressive cutaneous lesions in extremities in older men. Visceral organ involvement is rare in classic KS.[2]

In this study, a 72-year-old male classic KS patient with skin, gastrointestinal system, and lymph node involvement was discussed in terms of clinicopathologic features.

\section{Case Report}

A 72-year-old male patient observed for 10 years with a diagnosis of cutaneous classic KS was hospitalized 

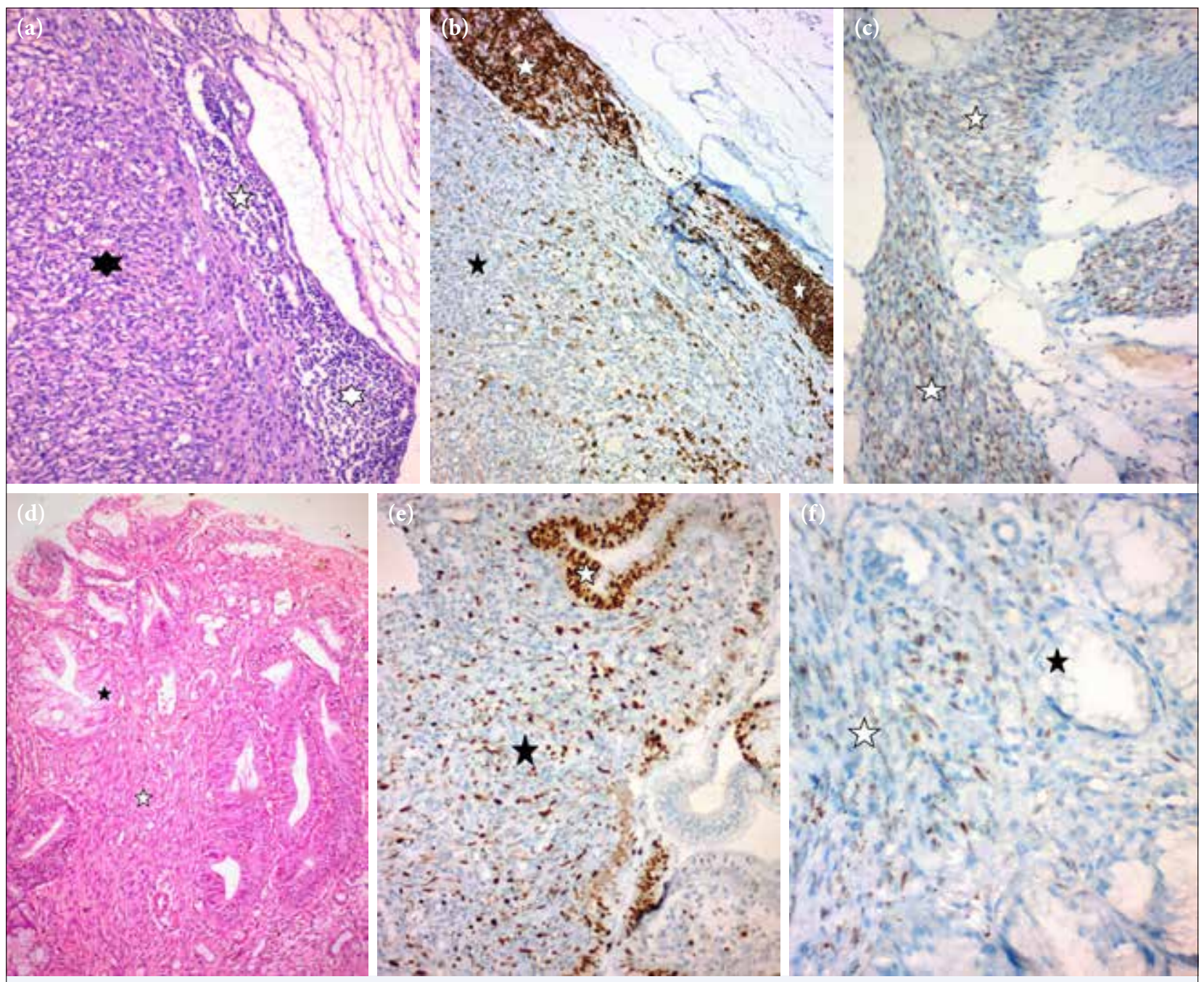

Fig. 1. (a) Neoplastic infiltration is observed, which removes nearly all the normal lymph node structure in lymphadenopathic Kaposi's sarcoma. Residual lymphoid tissue is seen in the subcapsular area (black asterisk). In some areas, typical nodular stage Kaposi sarcoma histology is observed (white asterisk) [A; H\&E, ×200]. (b) LCA monoclonal antibody in immunohistochemical assays facilitates the detection of residual lymphoid tissue. (c) Kaposi's sarcoma tumor cells show positive immunoreactivity with HHV-8 (LNA-1) (white asterisk) [B-C, BSA-DAB, BX200, CX400]. (d) Spindle cell proliferation infiltrating stroma (white asterisk) under superficial glandular epithelium (black asterisk) in the histology of the lesion observed as hemorrhagic nodule endoscopically in the postbulbular region $(D ; H \& E, \times 100)$. Immunohistochemical studies have shown that the proliferative index of tumor cells with Ki67 monoclonal antibody is high (black asterisk) (e) and that these cells are immunopositive with HHV-8 (LNA-1) antibody (f) (white asterisk) [E-F, BSA-DAB, $\times 400]$.

for lower respiratory tract infection. It was learned that the patient had been working for 20 years in a coal mine and was retired. The patient was on steroids for 6 months due to interstitial lung disease. On systemic examination, purple papular lesions were observed on the skin and bilateral cervical, preauricular, postauricular, and supraclavicular regions, the largest $3 \mathrm{~cm}$ in diameter lymphadenopathies were found. On laboratory examination, leukocytosis in peripheral blood and Pseudomonas bacteria in sputum culture were found.
The patient was found to be HIV-negative. On endoscopy, a hemorrhagic, hyperemic nodular lesion was found in the postbulbar region. Diagnostic biopsies were taken from lesional skin, duodenum, and lymph node regions. Histopathologic examination showed infiltrative, extravascular erythrocyte-bearing spindle cell proliferation, which removed the usual tissue in all three biopsy specimens. Typical and atypical mitotic figures were present in the tumor. In neoplastic cells, diffuse immunoreactions with CD31, CD34, vimen- 
Table 1 Histopathologic differential diagnosis of lymphadenopathic Kaposi sarcoma

Lesion

Reactive/Benign

Vascular transformation of lymph node sinuses

HIV linfadenitis-associated vascular hyperplasia, pattern C

Cellular hemangioma

Hemorrhagic spindle cell tumor with "amianthoid" fibrils

Inflammatory pseudotumor

Basillar angiomatosis

Castleman lymphadenopathy, hyalin-vascular type

Potential Malignancy/Malignant

Hemangioendothelioma

Angioimmunoblastic lymphadenopathy

Angiosarcoma

Lymphangiosarcoma

It should be considered that HHV-8 coinfection is frequent in HIV lymphadenitis and multicentric Castleman cases.

tin, and HHV-8 (LNA-1) were detected (Fig. 1). Histopathologic findings, skin lesion, nodule/tumor stage $\mathrm{KS}$, lymph node, and duodenal biopsy specimens were evaluated for KS involvement.

\section{Discussion}

Classic type $\mathrm{KS}$ is a vascular proliferative disorder characterized by moderate progression of multiple cutaneous lesions generally in middle-advanced aged male patients. Unlike other clinical forms of KS, visceral organ involvement is rare. Although the treatment approach varies according to the patient's clinical stage, surgery, cryotherapy and radiotherapy are recommended for localized disease. Systemic chemotherapy and/or interferon-alpha therapy can be used in advanced stage disease.[2]

Lymphadenopathic KS is a symptomatic manifestation of clinical lymph node enlargement resulting in neoplastic infiltration of KS in single or multiple lymph nodes. In classic KS, lymph node involvement is rare. In an epidemiological study in Morocco, the visceral organ involvement rate was $16 \%$ in classic KS cases and about half of these cases were lymphadenopathic KS cases.[3] Lymphadenopathic KS can also be seen in patients without skin involvement. In some cases, the clinical picture is associated with systemic symptoms, suggesting extensive lymphadenopathy and lymphoma. Histopathologic differential diagnoses of lymphadenopathic KS include benign and malignant soft tissue lesions with vascular and spindle cell compartments (Table 1).[4-6]

In classic KS, gastrointestinal system involvement is the second most common extra cutaneous localization after lymph node involvement. The colon, small intestine, and stomach are among the most common intestinal localizations. Lower gastrointestinal system KS may cause bleeding, perforation, and occasionally obstruction. Upper gastrointestinal system KS cases are usually asymptomatic and sometimes can be detected with hemoptysis.8 Gastrointestinal KS differential diagnosis includes a wide range of diseases. It can be confused clinically/endoscopically with nonneoplastic lesions such as ulcerative colitis, amebiasis, and conditions with massive lesions with spindle cell, vascular-rich tumors.[5]

Classic KS is generally characterized by slow-onset cutaneous lesions, and the mortality rate varies between $0.6 \%$ and $9 \%$ in various series. However, in older patients, many factors (i.e., chronic systemic diseases, immunosuppressive treatments, severe infections) that disrupt the general condition of the individual may reveal visceral organ involvement. It has been reported that cutaneous or visceral KS develops during immunosuppressive treatment regimens among individuals without a history of KS. KS may develop during rituximab treatment, which is used for severe autoimmune disorders, chronic inflammatory disorders, and prevention of organ rejection, and may regress following its cessation.[7-8] Some important data suggest that steroids substantially increase KS-related mortality, particularly in patients with AIDS.[9] Gastrointestinal KS may develop in patients with ulcerative colitis who are on steroid therapy.[10] Therefore, it is recommended to particularly investigate the lymph nodes and gastrointestinal system in classic KS cases. It should be kept in mind that classic KS cases with visceral organ involvement may show a more aggressive course.[6]

Our case, similar to the general characteristics of classic KS, was of advanced age and male sex. For many years, localized cutaneous KS was diagnosed without visceral organ involvement. We think that the development of visceral organ involvement in our case was highly likely due to steroids used in the treatment of interstitial lung disease and severe pulmonary infection. Our idea is supported by several previous studies reporting that steroids and other immunosuppressive agents cause the emergence of de novo KS lesions or the progression of pre-existing KS lesions.

In our case, upper gastrointestinal system KS involvement was asymptomatic and detected during endoscopic examination in accordance with the literature. It has been reported that systemic involvement may occur in classic KS cases, rarely without cutaneous lesions. A more detailed clinical examination of visceral organ involvement in classic KS cases may 
provide more accurate information in determining the frequency of systemic involvement of this disease.

\section{Conclusion}

The clinical behavior of KS, defined as a low-grade vascular tumor, may vary according to the epidemiological type and host's general status. Secondary diseases and therapies that suppress the immune system in classic KS cases can change the course of disease and may lead to the development of lesions with unusual localizations and atypical morphology. Therefore, in addition to recognizing the typical histologic features of KS in pathological terms, it is important to know the characteristics of the lesions during activation and regression and to demonstrate the presence of KSHV/ HHV-8 in tumor cells for definitive diagnosis.

\section{Disclosures Statement}

The authors declare no conflicts of interest.

Ethics Committee Approval: This study was conducted inaccordance with local ethical rules.

Peer-review: Externally peer-reviewed.

Conflict of Interest: None declared.

Authorship contributions: Concept - N. O. K; Design - N. O. K, F. B; Supervision - N. O. K, G. Y; Materials - N. O. K, B. D. G; Data collection \&/or processing - N. O. K, İ. E. B; Analysis and/or interpretation - N. O. K, Ş. O. Ö; Literature search - N. O. K, F. B; Writing - N. O. K, Ş. O. Ö; Critical review - N. O. K, F. B, G. Y, B. D. G, İ. E. B, S.. O. Ö.

\section{References}

1. Vincenzi B, D’Onofrio L, Frezza AM, Grasso RF, Fausti V, Santini D, et al. Classic Kaposi Sarcoma: to treat or not to treat? BMC Res Notes 2015;8:138.
2. Akasbi Y, Awada A, Arifi S, Mellas N, El Mesbahi O. Non-HIV Kaposi's sarcoma: a review and therapeutic perspectives. Bull Cancer 2012;99(10):92-9.

3. Errihani H, Berrada N, Raissouni S, Rais F, Mrabti H, Rais G. Classic Kaposi's sarcoma in Morocco: clinicoepidemiological study at the National Institute of Oncology. BMC Dermatol 2011;11:15.

4. Ioachim HL, Medeiros LJ. Ioachim's Lymph Node Pathology. 4th ed. Philadelphia: Lippincott Williams \& Wilkins; 1995. p. 570-87.

5. Pantanowitz L, Dezube BJ. Kaposi sarcoma in unusual locations. BMC Cancer 2008;8:190.

6. Sánchez-Peña P, Romero-Guadarrama MB, AguirreGarcía J. Diseases associated with HIV infection: study of biopsies and surgical resection specimens at a large general hospital in Mexico City. Ann Diagn Pathol 2009;13(3):162-7.

7. Clifford KS, Demierre MF. Progression of classic Kaposi's sarcoma with rituximab. J Am Acad Dermatol 2005;53(1):155-7.

8. Brambilla L, Tourlaki A, Genovese G. Iatrogenic Kaposi's Sarcoma: a Retrospective Cohort Study in an Italian Tertiary Care Centre. Clin Oncol (R Coll Radiol) 2017;29(10):e165-71.

9. Chabria S, Barakat L, Ogbuagu O. Steroid-exacerbated HIV-associated cutaneous Kaposi's sarcoma immune reconstitution inflammatory syndrome: 'Where a good intention turns bad'. Int J STD AIDS 2016;27(11):1026-9.

10. Svrcek M, Tiret E, Bennis M, Guyot P, Fléjou JF. KSHV/HHV8-associated intestinal Kaposi's sarcoma in patient with ulcerative colitis receiving immunosuppressive drugs: report of a case. Dis Colon Rectum 2009;52(1):154-8. 